\title{
SERUM IODINE OF EUTHYROID SUBJECTS TREATED WITH DESICCATED THYROID ${ }^{1,2}$
}

\author{
By DOUGLAS S. RIGGS, EVELYN B. MAN, AND ALEXANDER W. WINKLER \\ (From the Laboratory of the Fairfield State Hospital, Newtown, Connecticut, and the Departments \\ of Psychiatry and Internal Medicine, Yale University, School of Medicine, \\ and the New Haven Hospital and Dispensary, New Haven)
}

(Received for publication March 19, 1945)

The changes in serum iodine and basal metabolic rate which occur when myxedematous patients are treated with desiccated thyroid have been reported in the preceding paper (1). Many euthyroid subjects differ from myxedematous patients in their ability to tolerate comparatively large amounts of dried thyroid without manifesting significant signs or symptoms of hypermetabolism (2). In the present paper, the influence of thyroid feeding on serum iodine as well as on basal metabolism of euthyroid subjects is described, and the mechanism of euthyroid tolerance to large doses of desiccated thyroid is discussed.

\section{MATERIALS AND METHODS}

Data from 2 groups of patients are presented.

The first group consisted of 4 female schizophrenic patients in the Fairfield State Hospital, who were chosen for their willingness to cooperate in an extended experimental study. None of the 4 showed any evidence of thyroid dysfunction, and all were in reasonably good physical condition. The medical and psychiatric status of each patient is summarized at the end of the paper.

The basal metabolic rate of each patient was determined once a week. Duplicate 8-minute tests were performed using the Sanborn Motor-Grafic metabolism apparatus. Patients were required to rest in bed for $1 \frac{112}{2}$ hours before the test. The lower of the 2 rates determined during each test period was used rather than the average of both readings. Basal metabolic rates of patients $\mathrm{Pa}$., Ve., and Vi. were usually quite satisfactorily measured, those of Wi. less accurately. Body weight, pulse rate, oral temperature, and blood pressure were measured under basal conditions. Concentrations of precipitable iodine and of filtrable iodine in serum from venous blood were determined each week by methods previously described $(3,4)$. If the serum could not be precipitated at once it was placed in a securely-stoppered

1 This investigation was aided by a grant from the Fluid Research Funds of the Yale University School of Medicine.

2 A preliminary report of this work has appeared in the Proceedings of the American Society for Clinical Investigation in the Journal of Clinical Investigation, 1944, 23, 931.
$50 \mathrm{ml}$. Erlenmeyer flask and stored in a quick-freezing chamber. When frozen sera were analyzed, great care was exercised to agitate the thawed serum thoroughly before taking duplicates for analysis, because during freezing the serum proteins are concentrated at the bottom of the flask. The accuracy of serum iodine determination was about \pm 0.5 gamma per cent. Serum total proteins were determined each week.by the falling drop method of Barbour and Hamilton (5). Serum total cholesterol and serum titrated fatty acids were determined at monthly intervals by methods previously described $(6,7)$. Complete blood counts were done every 2 or 3 weeks throughout the course of the investigation. The first 5 weeks of the study of each patient constituted a control period. U.S.P. desiccated thyroid * was then given daily in powdered form as a suspension in a small amount of water under the close supervision of a graduate nurse or of an experienced head attendant. The dose was increased stepwise at 4-week intervals from 3 to $6,10,15$, and finally to 20 or 25 grains per day. The highest dose was maintained for 6 to 8 weeks, and then abruptly discontinued. Thereafter the patients were followed until no further significant changes in serum iodine or basal metabolic rate were observed. Minor individual variations from the program outlined above appear in Figure 1.

The second group consisted of 9 ambulatory patients followed for considerable periods of time in the metabolism clinic of the New Haven Hospital. The clinical status of each of these patients is described in the legend of Figure 2. Determinations of basal metabolic rate and serum total iodine were made at approximately monthly intervals. Barring unusual iodine intake, serum total iodine does not differ significantly from serum precipitable iodine. The dose of thyroid and the duration of treatment of each patient are indicated in Figure 2. Data concerning the metabolic response of 3 of these subjects to various doses of thyroid have already been published (2).

\section{RESULTS}

\section{A. Fairfield State Hospital Patients}

The changes in basal metabolic rate, serum precipitable iodine and other significant variables before, during, and after administration of desiccated thyroid to the 4 schizophrenic patients are depicted in Figure 1. In general the signs and

\footnotetext{
* Parke-Davis Brand.
} 


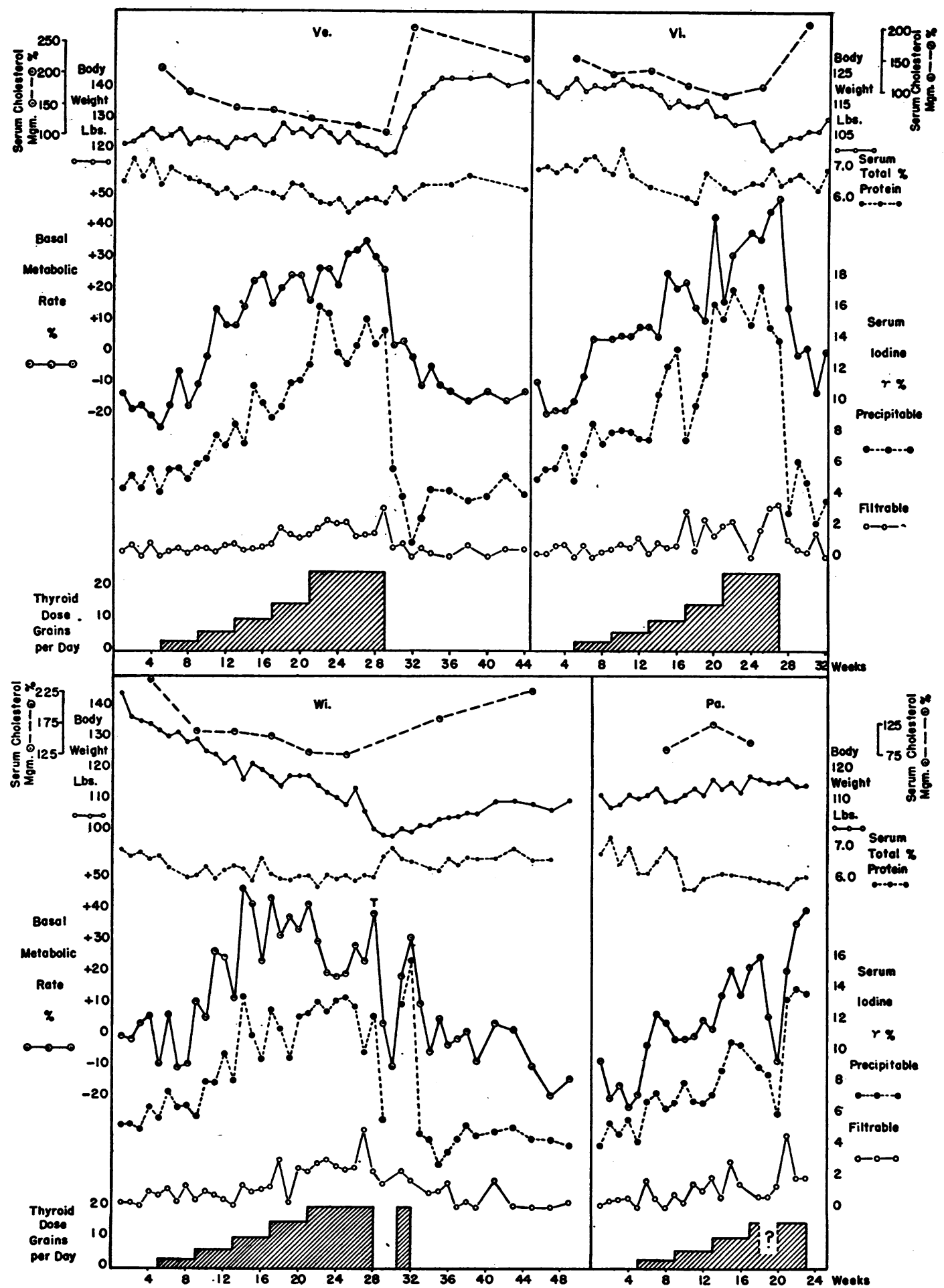

Fig. 1. The Effect of Large Doses of Desiccated Thyroid on Serum Iodine, Basal Metabolic Rate, and Other Variables in the Euthyroid Schizophrenic Patients

The significant changes depicted in these graphs are discussed in the text. Each point represents a different determination. The basal metabolic rate of Wi. on the twenty-eighth week (denoted by " $T$ " in the graph) is probably too high since the patient had an oral temperature of $100.1^{\circ} \mathrm{F}$. when the rate was determined. The question mark on the graph of thyroid dose for $\mathrm{Pa}$. indicated that for a 2 -week period the dose of thyroid was indeterminate, but probably small. 


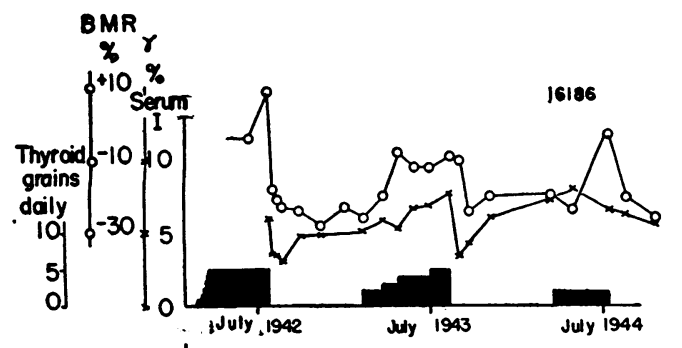

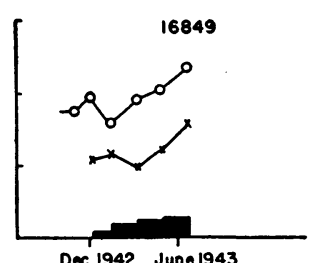

Dec.1942 June 1943
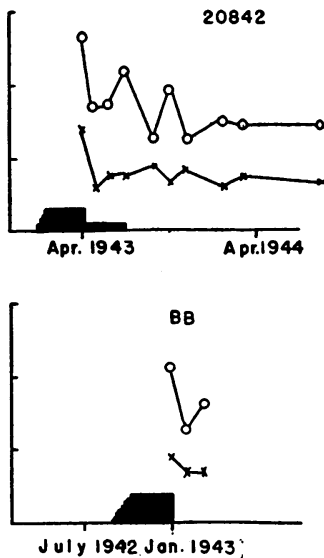
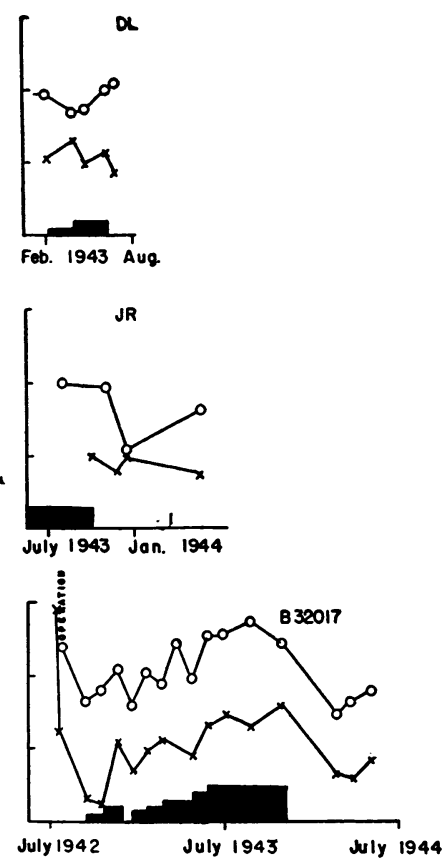

Fig. 2. Basal Metabolic Rate and Serum Iodine in Various Nonmyxedematous Subjects Receiving Thyroid Medication at Various Levels of Dosage for Extended Periods of Time

In general, metabolic rate and serum iodine are well correlated with one another but poorly correlated with the level of thyroid dosage. The differences in response to thyroid among various subjects should be noted.

The reactions to thyroid medication of the basal metabolism alone of the 3 subjects on the extreme left (16186, A13481, and A44014) have been described elsewhere and their case histories summarized there (2).

J. R. and B. B. were normal subjects who had been receiving thyroid for some time prior to this study solely because of initially low basal metabolic rates. Case 16849 and D. L. were normal subjects with an initial slight depression of metabolism who were deliberately placed on increasing doses of thyroid. Case 20842 underwent a subtotal thyroidectomy in 1942 which was followed by hypoparathyroidism and a low basal metabolic rate. At the time of the first observation by us (April, 1943), she had been on 3 grains of thyroid daily for some time, and was very nervous. Thyroid medication was first reduced to 1 grain a day and then omitted altogether. No clinical signs of thyroid deficiency ever developed. She was treated throughout with calcium and Hytakerol. Case B32017 represents a somewhat similar case followed from time of operation. Thyroid medication was given soon after operation because of definite symptoms of hypothyroidism. The dose was gradually raised to 5 grains daily, then stopped altogether. No symptoms of hypothyroidism developed, although both iodine and metabolism dropped.

symptoms of the patients during the period of excessive thyroid medication were those of a mild to moderate degree of hyperthyroidism, without exophthalmos or palpable enlargement of the thyroid gland.

With thyroid medication, the metabolic rates rose somewhat, but were not consistently maintained above +15 per cent until the dose was increased to 10 grains per day or more. With further increase in dosage, metabolic rates frequently rose above +30 per cent, but never exceeded +50 per cent. With discontinuance of thyroid treatment the metabolic rates fell rapidly at first. Subsequently, however, the fall was much less rapid, so that the decrease in basal metabolic rate approximated the logarithmic decay curve noted by previous observers $(8,9)$.

The serum precipitable iodine values for the 4 patients during the initial 5-week control period were well within normal limits. The serum iodine tended to rise following thyroid administration. As with the basal metabolic rate, however, frankly abnormal levels (above 8.0 gamma per cent) were not consistently maintained until a dose of 10 
grains per day was reached. Further increases in thyroid dose occasioned further increases in serum precipitable iodine (10). When thyroid treatment was discontinued the serum precipitable iodine fell so abruptly that within a single week normal or subnormal values were attained. By the end of the third or fourth week without thyroid, distinctly low values, comparable to those observed in untreated myxedema, were found. Thereafter the serum precipitable iodine again increased until normal levels were attained by the fifth week after cessation of medication.

The mean of 20 control determinations of serum filtrable iodine on the group of schizophrenic patients was 0.4 gamma per cent. The tendency of the serum filtrable iodine to rise when large doses of desiccated thyroid were administered is depicted in Figure 3. With cessation of treatment, the serum filtrable iodine rapidly reverted to a normal low level.

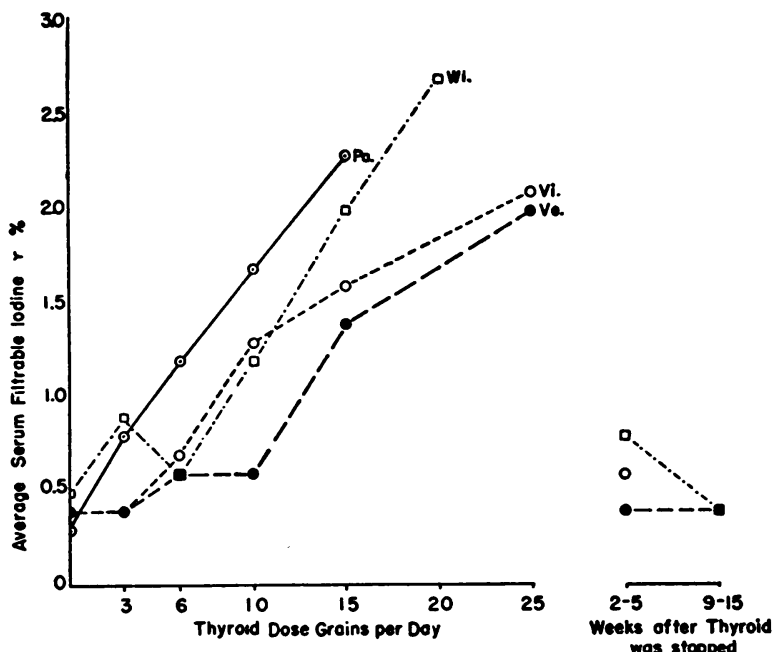

Fig. 3. The Effect of Large Doses of Desiccated Thyroid on Serum Filtrable Iodine in the Euthyroid Schizophrenic Patients

Each point represents the average of all determinations for a given patient on a given dose of thyroid. The larger the dose, the higher in general is the filtrable iodine of serum. After 2 to 5 weeks concentrations of filtrable iodine had returned to normal.

From initial normal values the serum cholesterol of Vi., Ve., and Wi. fell during thyroid treatment. $\mathrm{Pa}$. also had extremely low serum cholesterol values during treatment but unfortunately a cholesterol determination was not obtained during the control period. The greatest decrease was occasioned by the change from 0 to 3 grains per day, larger doses having comparatively much less effect. When thyroid administration was discontinued, the serum cholesterol concentration returned to normal.

Serum total protein decreased in response to the administration of thyroid. When thyroid treatment was stopped the serum protein tended to rise slowly toward the original level.

The influence of thyroid feeding on body weight in this group of patients was remarkably variable. Wi. lost weight during the whole period of medication but had already been losing weight during the control period. Vi. maintained weight on 3 and 6 grains daily but lost weight on larger doses. $\mathrm{Pa}$. and $\mathrm{Ve}$. showed no striking weight changes during the entire period of thyroid treatment. With discontinuance of thyroid medication the body weight tended to rise. The increase was most striking in Ve. who gained 25 pounds in 6 weeks, attaining a level considerably above her initial weight. None of the patients would admit any change in appetite during or following the period of thyroid feeding.

The changes in heart rate roughly paralleled the changes in basal metabolic rate. On the average a change of 10 per cent in the metabolic rate was associated with a change of 5 beats per minute in the heart rate. During the period of thyroid feeding the pulse pressures of all 4 patients rose considerably. For patients $\mathrm{Ve}$. and $\mathrm{Vi}$., the rise was due to a simultaneous increase in systolic and decrease in diastolic pressures. For patient $\mathrm{Pa}$., the pulse pressure rise was due almost entirely to a marked increase in systolic pressure, the diastolic pressure showing no significant trend. The reverse was true for patient Wi. In general there was a fairly high degree of correlation between the pulse pressure and pulse rate. Such minor fluctuations in body temperature as occurred during the study appeared to be due to random variation and could not be correlated with basal metabolic rate or dose of thyroid. The average oral temperature under basal conditions for all readings on all patients was very close to $98.0^{\circ} \mathrm{F}$. Of 139 temperature readings only 8 fell outside the range of $97.0^{\circ} \mathrm{F}$. to $99.0^{\circ} \mathrm{F}$. The erythrocyte count and hemoglobin of $\mathrm{Pa}$. and $\mathrm{Ve}$. showed no significant variations during the course of the study. The other 2 patients developed a slight anemia during the period of thyroid feeding. Wi.'s hemoglobin dropped from an average of 11.6 grams per $100 \mathrm{ml}$. on 0 to 6 grains of thyroid per day, to an average of 10.4 grams per $100 \mathrm{ml}$. on 15 to 20 grains of thyroid per day. The corresponding 
change for $\mathrm{Vi}$. was from 12.9 grams per $100 \mathrm{ml}$. to 11.6 grams per $100 \mathrm{ml}$. The red cell count fell commensurately. Although the total leucocyte count of Wi. underwent considerable fluctuation during the experimental period, no definite trend was evident. For the other 3 patients a distinct drop in the total leukocyte count during thyroid medication occurred. The lowest total white counts for Ve., Pa., and Vi. were $3,300,4,300$ and 4,700 cells per cubic millimeter, respectively. This fall in total white count was due almost entirely to a decrease in the absolute number of neutrophils. Although there was a relative lymphocytosis, the absolute number of lymphocytes was remarkably constant. After thyroid was stopped the hemogram tended to return to normal.

\section{B. New Haven Hospital Patients}

Figure 2 illustrates the effects of smaller doses and smaller increments of dosage continued over longer periods of time than those used in the schizophrenic patients of Figure 1. Changes of serum iodine and of basal metabolism in 9 nonmyxedematous patients on various levels of thyroid medication are charted. In general the results were quite similar to those shown in Figure 1. Basal metabolism followed serum iodine, while both were poorly correlated with the dose of thyroid in most subjects. Different subjects exhibited tolerance in varying degrees; 16186 failed to respond to 3 grains, but responded to 5 grains daily; A13481 tolerated 8 grains daily without significant change; 16849 tolerated only 2 grains daily and exhibited a distinct response to 3 grains. B32017 responded to 2 grains shortly after operation, while still partially hypothyroid; later 3 grains daily were tolerated but a sustained response followed a daily dosage of $\mathbf{5}$ grains. Fall of serum iodine to subnormal levels upon abrupt cessation of medication, followed by spontaneous recovery, is well illustrated in case 16186 (1942 and 1943). Under comparable circumstances serum iodine also fell and recovered less dramatically in case A13481, and fell in case A44014.

\section{. DISCUSSION}

Except for exophthalmos and goiter, most features of naturally occurring Graves' disease were reproduced by the oral administration of dried thyroid in large doses. Basal metabolism and serum precipitable iodine were elevated, while serum cholesterol was depressed. The responses of body weight, heart rate, and blood pressure were similar to those of spontaneous hyperthyroidism. The changes in total leukocyte count and in the relative proportion of lymphocytes in Graves' disease were reproduced by thyroid medication.

The effect on the basal metabolism of doses of thyroid below 6 or 8 grains per day was exceedingly capricious. For instance, the metabolic rate of Vi. (Figure 1) rose markedly on 3 grains of thyroid daily but failed to change significantly when the dose was doubled. The rate of A13481 was unaltered by a gradual increase of thyroid to 8 grains daily, while 16849 responded to an increment of dose as small as 1 grain daily. With increase of dosage above 10 grains daily the metabolic rate always rose somewhat above normal, but the relationship between dose and response was unpredictable and highly irregular. These observations confirm the experience of previous workers $(2,11,12)$.

In contrast to the poor correlation between thyroid dose and metabolic rate was the excellent correlation under most circumstances between serum precipitable iodine and metabolic rate. A stable serum iodine was regularly associated with failure of response of basal metabolism to thyroid medication (Figure 2). Whenever the metabolic rate increased significantly, there was a parallel increase in serum iodine. Each point in Figure 4 represents 1 pair of values of those 2 variables derived from the 4 experiments of Figure 1. Values after discontinuance of thyroid medication have been excluded. The distribution is curvilinear. Assuming the relationship to be logarithmic, as indicated by the solid lines in the graph, the coefficients of correlation between the basal metabolic rate and the logarithm of the serum precipitable iodine were $+0.93,+0.92,+0.88$, and +0.81 for $\mathrm{Pa}$. , Ve., Vi., and $\mathrm{Wi}$., respectively. This high degree of correlation indicates a close functional relationship, but by no means proves that the relationship is actually a logarithmic one. Indeed, if the same logarithmic relationship is assumed to extend below the normal range into the zone of values associated with hypothyroidism, the theoretical curve fails utterly to coincide with the observed values.

With increasing thyroid dosage, there was almost no lag of the rise in the basal metabolism behind that of the serum iodine. This is shown in 


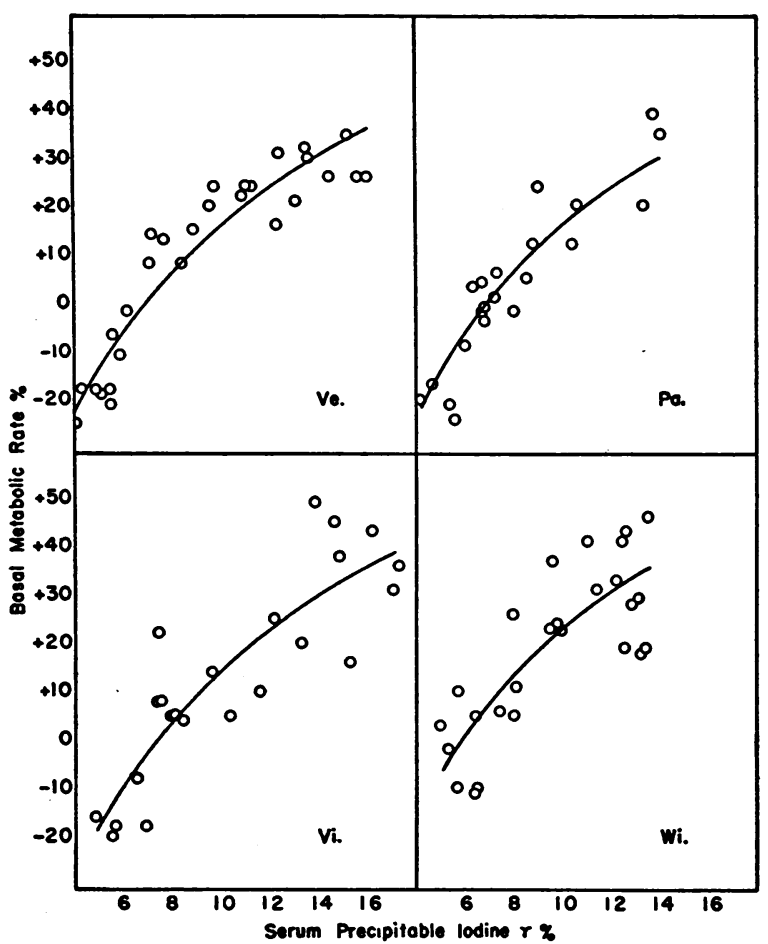

Fig. 4. Correlation between Serum Precipitable Iodine Basal Metabolic Rate in Four Euthyroid Schizophrenic Patients

The points represent individual determinations of serum precipitable iodine and corresponding values of basal metabolic rate. Values determined after thyroid was discontinued are omitted for reasons discussed in the text. The solid lines are the regression curves obtained when basal metabolic rate is assumed to be a function of the logarithm of the serum precipitable iodine.

Figure 5 , in which changes in serum iodine and in metabolic rate of the 4 schizophrenic patients following an increase in thyroid dose have been plotted against time. The curves represent mean values for all doses. On the average, the serum iodine had almost attained its maximum level 1 week following a change in dose, while the basal metabolic rate required 2 weeks to reach its maximum level. This difference is of doubtful significance.

This apparent parallelism in Figure 5 may conceal a true lag in metabolic response behind increase in serum iodine concentration, since dosage was being progressively increased. With cessation of thyroid dosage, certainly, serum iodine fell much more abruptly than did the basal metabolic rate. In fact all correlation between the 2 vari- ables was lost during the few weeks just after abrupt withdrawal of medication. Had these points been included in Figure 4, the correlation between serum iodine and basal metabolic rate would have been considerably less exact. Whereas the serum precipitable iodine of $\mathrm{Wi}$. and Ve. fell to a low point 3 weeks following withdrawal of thyroid, the basal metabolic rates did not reach their lowest post medication values for 9 to 15 weeks (Figure 1 ). The serum iodine of Vi. dropped to a definitely subnormal value by the first week after interruption of medication, while the simultaneous basal metabolic rate was still +15 per cent (Figure 1). Decline of the serum iodine without comparable fall of the basal metabolic rate following sudden interruption of thyroid medication also appeared in cases 16186 , A13481, and A44014 (Figure 2). A similar lag of the metabolic response behind the serum iodine

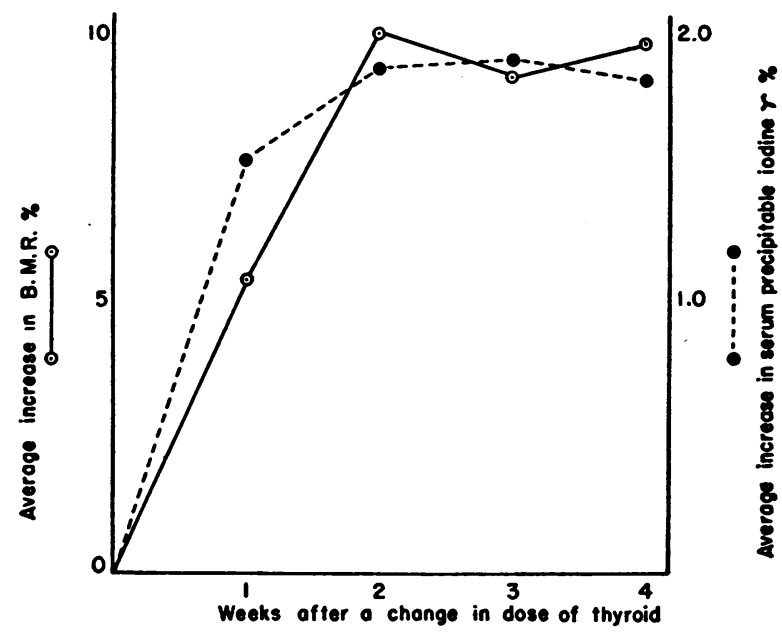

Fig. 5. Parallelism bteween the Rate of Change of Serum Precipitable Iodine and of Basal Metabolic Rate after an Increase in Thyroid Dose

With increasing thyroid dosage there is almost no lag in the rise in the basal metabolism behind that of the serum iodine. This contrasts with the marked lag of basal metabolism behind serum iodine with abrupt discontinuance of thyroid medication (Figures 1 and 2).

The increase in serum precipitable iodine or in basal metabolic rate occasioned by an increase in the dose of thyroid has been plotted against the time elapsed since the change in dose. The origin corresponds to the last value obtained while the previous dose was being administered. The points represent average values for increasing thyroid dosage in all 4 schizophrenic patients and for all increments of dose. 
concentration has been observed following thyroidectomy (patient B32017, and Figure 2 of the preceding paper) and following discontinuance of thyroid medication in patients with myxedema (1).

Presumably the lag of metabolic response behind serum iodine means that the thyroid hormone tends to disappear from the serum before it disappears from the tissues. The subnormal values of the serum iodine developing immediately after discontinuance of thyroid medication suggest not only inhibition by the medication of normal activity of the thyroid gland, but also a distinct delay in resuming its usual full activity. Persistence of an excessive rate of destruction of thyroid hormone may be a contributory factor.

Possible mechanisms of euthyroid tolerance to thyroid medication. In discussing possible reasons for the relative insensitivity of euthyroid subjects to desiccated thyroid, a group of workers (2) suggested that "the non-myxedematous subject possesses the ability, wanting in patients with myxedema, to inactivate thyroid substance and intravenous thyroxine." Perhaps it would have been more conservative to have suggested merely that this ability to inactivate thyroid hormone is very much greater in euthyroid subjects than in those with myxedema. The data of the present paper provide further support for this hypothesis, in that they disprove the existence of any decreased sensitivity of the tissues of the euthyroid subject to the thyroid hormone. The metabolic rate rises at least as much, for a given rise in serum iodine, in euthyroid as in myxedematous subjects (Figure 6). Of course a much larger dose of thyroid is required to produce a gross rise of serum iodine in the euthyroid subjects. The rise in the metabolic rate to supranormal levels in euthyroid subjects, induced by huge doses of desiccated thyroid was associated with a rise in serum iodine of about the same degree as that found in spontaneous hyperthyroidism of comparable severity. Whenever the metabolic rate was unaffected by thyroid medication, the serum iodine was also unchanged. It has been pointed out before (2) that impaired absorption of thyroid substance from the gastrointestinal tract by euthyroid subjects is an unsatisfactory explanation of their greater tolerance, since they are also comparatively insensitive to thyroxine administered parenterally. Also difficulty in absorption is never seen either in spontaneous or induced myxedema, so that it is highly improbable that euthyroid subjects without intestinal defects are peculiar in their absorption of thyroid hormone. Some storage of the hormone in the thy-

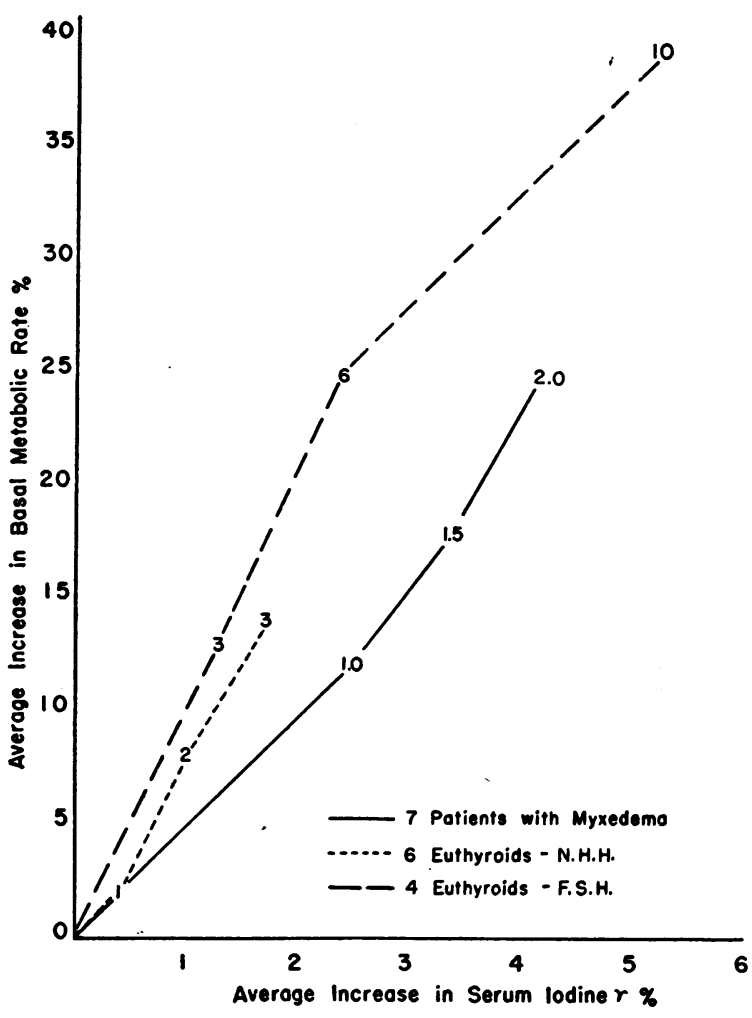

Fig. 6. Relationship Between the Increase of Serum Precipitable Iodine and of Basal Metabolic Rate of Myxedematous Patients and of Euthyroid Subjects Given Desiccated Thyroid

The increase in basal metabolic rate above the average premedication level is plotted against the corresponding increase in serum precipitable iodine at various levels of thyroid dosage. The dose in grains per day is indicated by the figures on the curves. The curve for the patients with myxedema was calculated from data reported in the preceding paper (1). Data from the 2 groups of euthyroid subjects reported here are plotted separately, the New Haven Hospital patients being indicated by short dashes, the Fairfield State Hospital patients by long dashes. Each point is the average for all patients in a given group on a given dose of thyroid.

Note that the basal metabolic rate rises at least as much per unit increase in serum iodine in the euthyroid subjects as in those with myxedema, but that a much larger dose of thyroid is required. 
roid gland as colloid is possible, but does not explain the indefinite persistence of tolerance. Enlargement of the thyroid gland was never noted even after weeks of administration of huge doses of thyroid substance to the schizophrenic subjects, although the gland would have had to increase by several hundred grams had even half the administered hormone beer stored. Extensive temporary storage in the thyroid gland or elsewhere is also improbable because a low serum iodine develops during the period following cessation of medication, and because subsequently there is never evidence of excessive release of stored hormone.

Inhibition of hormone production by the thyroid gland apparently did occur, but this factor only partially explains euthyroid tolerance to thyroid medication. There is good experimental evidence that the administration of thyroid hormone to intact animals depresses the normal activity of the thyroid gland $(13,14)$. In the present study of human subjects, the fall in serum iodine to very low levels when thyroid medication was abruptly withdrawn probably resulted from inhibition of normal production of hormone by the thyroid gland during the previous period of thyroid medication. Normal production of the hormone is, however, equivalent to not more than 3 grains of desiccated thyroid per day (1), so that complete cessation of endogenous production of hormone would account only for tolerance to this small daily dose. Were this the only mechanism concerned with the maintenance of tolerance, serum iodine and basal metabolism of all euthyroid subjects should respond to as much as 5 or 6 grains of thyroid daily. In point of fact, however, some normal subjects can tolerate as much as 8 grains daily without any significant alteration in serum iodine or metabolic rate (Figure 2).

A logical explanation of tolerance to amounts of desiccated thyroid in excess of 3 grains per day is that the normal thyroid gland can inactivate the exogenous hormone. This it might do by degrading active hormonal iodine to inorganic iodine, by a reversal of the reactions which, under normal circumstances, lead to the formation of hormone from inorganic iodine. The significant rise in the inorganic filtrable fraction of the serum iodine during massive thyroid feeding (Figures 1 and 3 ) would lend support to this hypothesis. It is not certain that all the inactivation takes place in the thyroid gland. Any large measure of inactivation anywhere else, however, would imply a fundamental difference between the tissues of euthyroids and the tissues of myxedematous patients with respect to their ability to dispose of thyroid hormone, since the only known difference between adequately-treated myxedematous patients and euthyroid subjects is the presence of functional thyroid tissue in the latter.

There is suggestive evidence that tolerance to desiccated thyroid may require considerable time for full development. Many euthyroid patients experience a sharp rise in metabolic rate for a few weeks subsequent to an increase in thyroid dose, but if the dose is maintained for a longer period of time the metabolic rate tends to drift downward towards its original level (2). Any latency in the development of tolerance in the various patients described in this paper would be partially masked by the gradual step-wise manner in which the dose was increased. Three of the 4 schizophrenic patients, while on the highest dose of thyroid for 6 to 8 weeks, showed no clear-cut evidence of a decreasing response. However, for 2 of the patients there was some suggestion of a partial loss of tolerance during a lapse in thyroid treatment. $\mathrm{Pa}$. had been started on 15 grains of thyroid per day, but after 1 week at this level the dosage was markedly reduced. Due to a confusion in orders, during the next 2 weeks she received irregularly an unknown, but probably small amount of thyroid. With resumption of a regular dose of 15 grains per day, both serum precipitable iodine and basal metabolic rate rose to levels much higher than would have been expected from this patient's previous responses. Similarly, when patient Wi. was replaced on a dose of 20 grains per day after a lapse of $21 / 2$ weeks, the serum iodine and metabolic rate both rose to a higher point than during the previous weeks on the same dose. These observations indicate the need for further investigation of the time necessary for the development of tolerance.

The tolerance of non-myxedematous patients to thyroid medication probably accounts for the apparent impunity with which large doses of dried thyroid may be administered under many circumstances. Whether permanent ill effects may re- 
sult from long continued administration of thyroid substance to euthyroid subjects is not known. Our experiments have demonstrated only functional depression of the thyroid gland, while thyroid is being given and for a brief period after its withdrawal. This fact alone, however, suggests that long continuance of thyroid medication may not be wholly benign. On a priori grounds, caution is certainly indicated, especially in the continuous administration of thyroid to adolescents and to patients recovering from subtotal thyroidectomy.

\section{SUMMARY AND CONCLUSIONS}

1. Many euthyroid subjects are able to tolerate considerable amounts of desiccated thyroid without significant alteration in either serum precipitable iodine or basal metabolic rate.

2. The administration of larger quantities of dried thyroid to euthyroid subjects produces abnormal elevation of both serum iodine and basal metabolic rate and elicits most of the signs and symptoms of spontaneous hyperthyrodism, but the dose of thyroid required is usually much greater than is needed to produce an equivalent degree of hyperthyroidism in myxedematous patients.

3. When the administration of desiccated thyroid to euthyroid subjects is abruptly discontinued, the serum precipitable iodine frequently falls to abnormally low levels, probably indicating temporary inhibition of hormone production by the normal thyroid gland during, and for a brief period following, thyroid feeding.

4. Rapid and transient changes in serum precipitable iodine may occur without comparable fluctuations in basal metabolic rate. When alterations in the level of serum precipitable iodine are slower and more sustained, the lag in metabolic response is not as evident, and there is a high degree of correlation between the 2 variables.

5. The tissues of euthyroid subjects are at least as sensitive to thyroid hormone as are those of myxedematous subjects.

6. Degradation of active hormonal iodine to inorganic iodine by the normal thyroid gland is suggested as the most likely explanation of the tolerance of euthyroid subjects to amounts of desiccated thyroid in excess of 3 grains per day.

\section{PROTOCOLS}

$V e$. was a 33-year-old white female with schizophrenia, paranoid type, of at least 2 years' duration. Although correctly oriented and in good contact with her surroundings, she believed herself persecuted by creatures half man and half beast. She was highly co-operative. Her mental symptoms were uninfluenced by thyroid medication. She was tall and thin without significant physical abnormalities except for vasomotor instability characterized by marked flushing of the skin of face, neck, and arms. Blood pressure was 100/60. On 6 grains of thyroid daily the vasomotor instability increased, and the patient fainted on 2 occasions. There was a slight tremor of the extended fingers. When larger amounts of thyroid were administered, the tremor became more pronounced and the vasomotor instability was further accentuated. The skin was warm and moist. The patient complained of fatigue and a "draggy" feeling. When thyroid was discontinued the tremor disappeared, the skin became drier, and the vasomotor instability decreased. Coincident with a marked gain in body weight, the patient became somewhat lethargic, but she never developed any signs or symptoms of myxedema.

Vi. was a 23-year-old white female with a 3-year history of schizophrenia, hebephrenic type. Before thyroid was administered she was extremely lethargic, withdrawn, and almost mute. She was hallucinated and grinned inappropriately. She was usually quite cooperative. During treatment with desiccated thyroid, the patient became more talkative and much less lethargic, assisting with ward work and taking long outdoor walks. With cessation of thyroid medication she reverted to her previous mute and disinterested condition. While this improvement in energy output may have been related to the induced hyperthyroidism, it should be noted that the patient had previously experienced similar, but apparently spontaneous, remissions. On physical examination prior to thyroid administration the patient was well developed and slightly obese with acne of the face and hirsutism of the face, back, and extremities. Blood pressure was 114/65. In response to thyroid, she developed tachycardia and a moist, warm skin with increased perspiration, but there was no definite tremor of the outstretched fingers. On 25 grains of thyroid daily, she complained of fatigue, and slight dyspnea on exertion. Observations during the postmedication period were interrupted when the patient was transferred to another hospital 5 weeks after thyroid feeding was stopped.

$W i$. was a 54-year-old white female who had been hospitalized for 14 years because of schizophrenia, paranoid type, characterized by fixed delusions of grandeur. Although much deteriorated mentally, she was cooperative enough to serve as a subject in the present study. Her psychosis was unaffected by thyroid medication. The only abnormality found on physical examination prior to the administration of desiccated thyroid was slight narrowing, irregularity, and increased tortuosity of the retinal arteries. Blood pressure was $120 / 75$. An $x$-ray of the chest was negative. On 6 grains of thyroid daily, the pa- 
tient felt somewhat weak, and on 1 occasion had a fainting spell, with marked pallor of the face, and imperceptible pulse, but without loss of consciousness. On larger amounts of thyroid, a slight tremor of the extended fingers was noted, and on a few occasions there was transient ankle edema. After 7 weeks on 20 grains of thyroid daily the patient developed a fever of $102^{\circ} \mathrm{F}$. The total leukocyte count was 11,200 with 73 per cent neutrophils. The pulse was 130 per minute. Except for a red and swollen but non-tender area at the base of 1 finger nail, there was no evidence of infection. Thyroid was discontinued. Despite the administration of sulfadiazine, the temperature continued to be somewhat elevated during the next 4 days. A chest $x$-ray showed left ventricular hypertrophy, but was negative for peripheral lung pathology. The only subjective complaint was stiffness and soreness of the shoulders, which persisted for some time after the fever had subsided. Although the cause of the hyperpyrexia was not clear, it was probably not due to the thyroid medication, since the same dose was later resumed for 2 weeks without untoward consequences.

$\mathrm{Pa}$. was a 23-year-old white female who developed symptoms of schizophrenia, type undetermined, 3 years prior to admission. She was lethargic with brief periods of manic excitement, and claimed that she was infested by "bristle beetles." She was well oriented and quite cooperative, but resented hospitalization and made several attempts to escape. While thyroid was being administered, her comments and thinking appeared more coherent; this was thought to be only a temporary improvement unrelated to the thyroid medication. Physically she was well developed but thin and pale. Blood pressure was 100/60. Even on 15 grains of thyroid daily, tachycardia was the only physical sign of hyperthyroidism. Unfortunately the patient escaped from the hospital before the experimental program was completed.

\section{BIBLIOGRAPHY}

1. Winkler, A. W., Riggs, D. S., and Man, E. B., Serum iodine in hypothyroidism before and during thyroid therapy. J. Clin. Invest., 1945, 24, 732.

2. Winkler, A. W., Lavietes, P. H., Robbins, C. L., and Man., E. B., Tolerance to oral thyroid and reaction to intravenous thyroxine in subjects without myxedema. J. Clin. Invest., 1943, 22, 535.

3. Riggs, D. S., and Man, E. B., A permanganate acid ashing micromethod for iodine determinations. I. Values in blood of normal subjects. J. Biol. Chem., 1940, 134, 193.

4. Man, E. B., Smirnow, A. E., Gildea, E. F., and Peters, J. P., Serum iodine fractions in hyperthyroidism. J. Clin. Invest., 1942, 21, 773.

5. Barbour, H. G., and Hamilton, W. F., The falling drop method for determining specific gravity. J. A. M. A., 1927, 88, 91.

6. Man, E. B., and Peters, J. P., Gravimetric determination of serum cholesterol adapted to the Man and Gildea fatty acid method with a note on the estimation of lipoid phosphorus. J. Biol. Chem., 1933, $101,685$.

7. Bogdanovitch, S. B., and Man, E. B., The effects of castration, theelin, testosterone, and antuitrin-S on the lipoids of blood, liver, and muscle of guinea pigs. Am. J. Physiol., 1938, 122, 73.

8. Means, J. H., and Lerman, J., The curves of thyroxine decay in myxedema and of iodine response in thyrotoxicosis: their similarity and its possible significance. Ann. Int. Med., 1938, 12, 811.

9. Boothby, W. M., and Baldes, E. J., Some quantitative relationships of thyroxine calculated from its calorigenic action. J. Pharmacol. and Exper. Therap., 1925, 25, 139.

10. Farquharson, R. F., and Squires, A. H., Inhibition of secretion of thyroid gland by continued ingestion of thyroid substance. Tr. A. Am. Physicians, 1941, 56, 87.

11. Kunde, M. M., Studies on metabolism. VI. Experimental hyperthyroidism. Am. J Physiol., 1927, 82, 195.

12. Means, J. H., The Thyroid and Its Diseases. J. B. Lippincott Co., Philadelphia, 1937, p. 523.

13. Balasco, I. J., and Murlin, J. R. The effect of thyroxin and thyrotropic hormone on basal metabolism and thyroid tissue respiration of rats at various ages. Endocrinology, 1941, 28, 145.

14. Cameron, A. T., and Sedziak, F. A., The effect of thyroid feeding on growth and organ hypertrophy in adult white rats. Am. J. Physiol., 1921, 58, 7. 